OPENACCESS

International Journal of Environmental Research and

Public Health

ISSN 1660-4601

Article

www.mdpi.com/journal/ijerph

\title{
Verification of the Addiction Severity Index Japanese Version (ASI-J) as a Treatment-Customization, Prediction, and Comparison Tool for Alcohol-Dependent Individuals
}

Ayako Haraguchi ${ }^{1}$, Yasukazu Ogai ${ }^{1}$, Eiichi Senoo ${ }^{2}$, Satoru Saito ${ }^{3}$, Yoshihiro Suzuki ${ }^{4}$, Aihide Yoshino ${ }^{5}$, Aro Ino ${ }^{6}$, Kenji Yanbe ${ }^{7}$, Mitsuru Hasegawa ${ }^{8}$, Masaru Murakami ${ }^{9}$, Masanobu Murayama ${ }^{10}$, Toru Ishikawa ${ }^{11}$, Susumu Higuchi ${ }^{12}$ and Kazutaka Ikeda ${ }^{1} *$

1 Division of Psychobiology, Tokyo Institute of Psychiatry, 2-1-8 Kamikitazawa, Setagaya-ku, Tokyo 156-8585, Japan; E-Mails: haragu-a@prit.go.jp (A.H.); y-oogai@prit.go.jp (Y.O.)

2 Division of Social Psychiatry, Tokyo Institute of Psychiatry, 2-1-8 Kamikitazawa, Setagaya-ku, Tokyo 156-8585, Japan; E-Mail: senoo@prit.go.jp

3 Institute for Family Functioning, 2-14-6 Azabu Ju-ban, Minato-ku, Tokyo 106-0045, Japan; E-Mail: yamanaka@iff.or.jp

4 Wakamiya Hospital, 2-15-3 Yoshiwara, Yamagata 990-2451, Japan;

E-Mail: wakamiya@koutoku.or.jp

5 National Defense Medical College Hospital, 3-2 Namiki, Tokorozawa, Saitama 359-8513, Japan; E-Mail: aihide@me.ndmc.ac.jp

6 Dansyu no Ie Clinic, 217 Fujikata, Tsu, Mie 514-0815, Japan; E-Mail: aroino@za.ztv.ne.jp

7 Asahiyama Hospital, 4-3-33 Futagoyama, Chuo-ku, Sapporo, Hokkaido 064-0946, Japan; E-Mail: a-ikyoku@gray.plala.or.jp

8 Okabe Hospital, 15 Nagasaka-machi-chi, Kanazawa, Ishikawa 921-8114, Japan; E-Mail: hasegawamitsuru@okabe-net.jp

9 National Hospital Organization Ryukyu Hospital, 7958-1, Kin, Kin-Cho, Kunigami-Gun, Okinawa 904-1201, Japan; E-Mail: murakami@m.email.ne.jp

10 Akagi-Kohgen Hospital, Kitaakagiyama, Akagi-machi, Shibukawa, Gunma 379-1111, Japan; E-Mail: masamura@msa.biglobe.ne.jp

11 Tohokukai Mental Hospital, 1-8-7, Kashiwagi, Aoba-ku, Sendai, Miyagi 981-0933, Japan; E-Mail: tishikawa@tohokukai.com

12 National Hospital Organization Kurihama Alcoholism Center, 5-3-1, Nobi, Yokosuka, Kanagawa 246-0841, Japan; E-Mail: h-susumu@db3.so-net.ne.jp 
* Author to whom correspondence should be addressed; E-Mail: ikedak@prit.go.jp;

Tel.: +81-3-3304-5701 ext. 508; fax: +81-3-3329-8035

Received: 7 July 2009 / Accepted: 10 August 2009 / Published: 12 August 2009

\begin{abstract}
Objective: To demonstrate the usefulness of the Addiction Severity Index Japanese Version (ASI-J) in Japanese alcohol-dependent individuals. The ASI is a frequently used clinical and research instrument that measures severities in seven functional domains in people with substance abuse disorders. Methods: A total of 370 male inpatients with a history of alcohol dependence participated in the study. Forty-nine participants were excluded in the final analysis due to lack of reliability (i.e., patient misrepresentation or inability to understand). We used the ASI-J and a series of indexes that determined patient states during and post-treatment. Results: The correlations between ASI Composite Scores (CSs), which were calculated through a weighted formula and indicated the severity of each problem area, were significant but low in eight relations and not significant in 13 relations, indicating substantial independence of the problem areas. Significant differences were found in Family/Social CSs between abstinent and relapsed alcohol-dependent individuals. The questions of undesirable attitude were significantly related to the CSs of Employment, Drug use, Family/Social, and Psychiatric sections. Significant differences were observed in patient demographics, CS, and ASI Severity Rating (SR) and interviewer's subjective scoring between alcohol-dependent individuals and drug abusers. CSs in Japanese alcohol-dependent individuals were generally similar to corresponding CSs in individuals from other countries, with the exception of The Netherlands. Conclusions: This study demonstrated that the ASI-J is useful for understanding individual profiles of problems for each patient and planning customized treatment. The ASI-J served as a predictive tool for relapse and compliance to treatment afterward and was shown to be useful as a comparison tool in clarifying similarities and differences between substance abuser groups.
\end{abstract}

Keywords: addiction severity index; alcohol dependence; japanese

\title{
1. Introduction
}

The appearance of alcohol dependence and its related disorders is hypothesized to be attributable to a patient's biological basis combined with psychological and social factors [1,2] such that each patient with alcohol dependence has individually different issues. Addicted patients cannot be adequately characterized simply by measuring the nature, amount, and duration of their substance use [3]. Addiction-related problems are typically reasons for referral to addiction treatment, are often of greater concern to the patient than the substance use itself, and are usually important for deciding the setting and content of care [3]. Therefore, customizing treatment for individual patients according 
to their problems and motivation toward treatment is ideal. However, no prevailing methods exist, at least in Japan, to comprehensively reveal multidimensional patient states related to treatment.

In addition to the difficulty in revealing patient states, the outcome of alcohol dependence treatment is difficult to evaluate, partially because predicting patient compliance to treatment and risk of relapse is difficult. Tools predicting a patient's prognosis will enable treatment staff to provide additional and adequate care to the patient.

To clinically apply the results of substance dependence studies, tools for comparing patient groups are useful. For example, when medicine and treatment approaches are used in different patient groups, understanding the similarities and differences of patient features and their treatment environments is critical. The wide use of common tools enables clinicians and researchers to compare patients with different demographics, patients with different substance dependence, patients in different countries, and patients and healthy controls. Such common assessment tools are also useful to grasp the profiles of target patient groups and refine treatment modalities.

The Addiction Severity Index (ASI) [4] is an epochal interview developed to achieve the aforementioned goals. Currently, the ASI has reached its fifth version [5], and the instrument has been translated into over 20 languages [3]. The application of the ASI to alcohol-dependent individuals has been verified in various countries (The Netherlands, Switzerland, United States, Germany [1,5-9]), and the ASI has nearly achieved both reliability and validity, although some problems still exist [10].

The ASI is a semi-structured clinical research interview designed to assess problem severity in seven functional domains: Medical, Employment/Support, Alcohol use, Drug use, Legal, Family/Social relationships, and Psychiatric. The ASI provides two types of overall scores for respective problem areas to rate the severity of the problem [11], including the composite score (CS) and the severity rating (SR). The CS is an objective score calculated through a weighted formula designed to provide an equal contribution from each item. The SR is a relatively subjective score indicating the need for additional treatment in the specific area based on interviewer assessment. The ASI has a system to clearly indicate a change of two severity scores by administering it before and after treatment. The ASI Japanese version (ASI-J) was applied to patients with a history of drug abuse, and its reliability and validity have already been confirmed [12]; however, the ASI-J has not yet been applied to patients with a history of alcohol dependence.

In the present study, we investigated the usefulness of the ASI-J in alcohol-dependent individuals in Japan to determine $(i)$ its usefulness as a tool for understanding problem profiles for each patient, (ii) its usefulness as a tool to determine patient prognosis, and (iii) its usefulness as a common assessment tool for comparison studies.

\section{Methods}

\subsection{Participants}

A total of 370 male inpatients with a history of alcohol dependence participated in the study. The participants were recruited from nine nationwide hospitals for addiction treatment in Japan $(n=91$, National Hospital Organization Kurihama Alcoholism Center, Kanagawa; $n=55$, Wakamiya Hospital, Yamagata; $n=50$, Komakino Hospital, Tokyo; $n=42$, Mie Prefectural Mental Medical Center, Mie; 
$n=26$, Asahiyama Hospital, Hokkaido; $n=17$, Ishikawa Prefectural Takamatsu Hospital, Ishikawa; $n=14$, National Hospital Organization Hizen Psychiatric Center, Saga; $n=13$, Akagi-Kohgen Hospital, Gunma; $n=12$, Tohokukai Mental Hospital, Miyagi). Inpatients were provided an average of 80-day treatment programs (group meeting, alcohol education, family treatment programs, psychotherapy, and so on) after detoxification. After recovery from serious physical and mental instability (almost 1 month after hospitalization), informed consent was obtained from the subjects, excluding the patients who had serious cognitive impairment and psychiatric problems, and the ASI-J was administered by psychiatrists who were expert in alcoholism, carefully read the ASI manual (http://www.tresearch.org/resources/manuals/ASIQbyQGuide.pdf), and learned the interview methods by themselves. The average time required for administration was $30 \mathrm{~min}$. Inpatient subjects were requested to answer the questions during the 30 days prior to the start of inpatient treatment. Forty-nine participants were excluded from the final analysis because of lack of reliability (i.e., patient misrepresentation or inability to understand). Methamphetamine abuse data that were compared in the present study were mainly obtained from our previous study that standardized the ASI-J [12]. A total of 116 ASI-J samples with a history of methamphetamine and/or amphetamine abuse were collected from three hospitals and two recovery centers.

\subsection{Instruments}

We used two types of indexes, including the ASI-J and a series of indexes called the Treatment and Recovery of Alcohol-dependence that determined patient state during and post-treatment. The ASI provides two types of overall scores for each problem area: severity rating (SR) and composite score (CS) [11]. The SR indicates the severity of the problem on the basis of interviewer assessment during in-person interviews using subjective and objective data related to current and lifetime problems. The SR ranges from 0 to 9 points, with a higher score indicating greater problem severity. The CS in each problem area is not a rating but rather a mathematically calculated score based on patient responses to sets of items asking for patient behaviors during the 30 days prior to interview. The CS is calculated using a weighted formula designed to provide an equal contribution from each item and varies from 0 to 1 , with a higher score indicating greater problem severity.

The Treatment and Recovery of Alcohol-dependence indexes were administered by the attending physician or deputy at hospital discharge and 3 months and 1 year post-discharge. Each index included 8-18 items that assessed attitudes toward hospital treatment (i.e., Lack of cooperation, Lack of leadership, Rule breaking, Relapse, and Substance abuse), biochemical markers (i.e., glutamic oxaloacetic transaminase [GOT], glutamic pyruvic transaminase [GPT], $\gamma$-glutamyl transpeptidase $[\gamma-\mathrm{GTP}])$, the frequency of relapse after discharge from the hospital, and so on. With regard to scoring the undesirable attitudes toward treatment, the attending physician subjectively rated the five aforementioned items on a 3 -point scale $(1=\operatorname{good}, 2=$ normal, $3=$ bad $)$.

\subsection{Statistical Analysis}

First, independence of problem areas was examined by calculating the Pearson's product-moment correlation coefficient among individual CSs. Second, possibilities of predictive assessments of 
relapse using the ASI-J were analyzed by comparing CSs between abstinent and relapsed alcohol-dependent individuals via one-way analysis of variance (ANOVA). Associations between CSs and undesirable attitudes toward treatment were examined by the Pearson product-moment correlation coefficient. Third, significant differences in profiles between alcohol-dependent individuals and drug abusers were examined using the $\chi^{2}$ test. Comparisons of CS and SR between alcohol-dependent individuals and drug abusers were conducted using one-way ANOVA. With regard to comparisons of CSs in alcohol-dependent individuals among studies, Welch's $t$-test was conducted using DA Stat (Shinko Koeki, Tokyo, Japan). Fourth, internal consistency of each ASI area was examined using Cronbach's alpha value and the Pearson product-moment correlation coefficient between the CS and SR of each area. All analyses, with the exception of comparisons of CSs in alcohol-dependent individuals among studies, were performed with SPSS v. 12.0 for Windows (SPSS Japan, Tokyo, Japan).

Table 1. Correlation coefficients between each ASI CS in alcohol-dependent individuals $(n=321)$.

\begin{tabular}{|c|c|c|c|c|c|c|c|}
\hline $\begin{array}{c}\text { Composite } \\
\text { score } \\
\end{array}$ & Mean \pm SD & Employment & $\begin{array}{c}\text { Alcoho } \\
\text { l use }\end{array}$ & $\begin{array}{c}\text { Drug } \\
\text { use }\end{array}$ & Legal & $\begin{array}{c}\text { Family/Soci } \\
\text { al } \\
\end{array}$ & Psychiatric \\
\hline Medical & $0.24 \pm 0.30$ & $0.13^{*}$ & $-0.19^{* *}$ & 0.06 & 0.05 & 0.01 & 0.00 \\
\hline Employment & $0.54 \pm 0.28$ & & -0.06 & 0.04 & $0.12^{*}$ & 0.07 & -0.03 \\
\hline Alcohol use & $0.67 \pm 0.28$ & & & -0.01 & 0.02 & $0.16^{* *}$ & $0.11^{*}$ \\
\hline Drug use & $0.01 \pm 0.04$ & & & & 0.00 & $0.14^{*}$ & $0.23^{* *}$ \\
\hline Legal & $0.01 \pm 0.04$ & & & & & 0.02 & -0.07 \\
\hline Family/Social & $0.25 \pm 0.22$ & & & & & & $0.32^{* *}$ \\
\hline Psychiatric & $0.15 \pm 0.20$ & & & & & & \\
\hline
\end{tabular}

\section{Results}

\subsection{Independence of Each ASI Area: Usefulness in Measuring Multidimensional Problems}

The ASI was developed to independently evaluate each of seven problem areas. To assess the independence of the seven areas, the correlation between each CS was analyzed (Table 1). Significant but low correlations were noted in eight relations: between the Medical CS, Alcohol use CS, and Employment CS; between the Employment CS and Legal CS; between the Alcohol use CS, Family/Social CS, and Psychiatric CS; between the Drug use CS, Family/Social CS, and Psychiatric CS; between the Family/Social CS and Psychiatric CS. The Psychiatric CS was significantly related with CSs in three areas. No significant correlations were observed in the other 13 relations. These results indicate substantial independence of the problem areas, with the exception of the Psychiatric CS. 


\subsection{Prediction of Prognoses}

3.2.1. Association between CSs and attitudes toward treatment in alcohol-dependent individuals

Items of undesirable attitude toward treatment were related to CSs of Employment, Drug use, Family/Social, and Psychiatric areas at the start of hospitalization (Table 2). The total points of undesirable attitude had weak but significant relationships with the CSs of Drug use, Family/Social, and Psychiatric (0.12-0.13). The Drug use CS was moderately related to substance abuse (actual relapse) during hospitalization (0.41).

Table 2. Correlation coefficients between CSs and attitudes toward treatment in alcohol-dependent individuals.

\begin{tabular}{|c|c|c|c|c|c|c|c|}
\hline $\begin{array}{c}\text { Attitude } \\
\text { toward treatment }\end{array}$ & Medical & Employment & $\begin{array}{c}\text { Alcohol } \\
\text { use }\end{array}$ & $\begin{array}{c}\text { Drug } \\
\text { use }\end{array}$ & Legal & Family/Social & Psychiatric \\
\hline Lack of cooperation & 0.016 & $0.119^{*}$ & -0.069 & 0.053 & 0.028 & 0.055 & $0.113^{*}$ \\
Lack of leadership & -0.024 & 0.065 & -0.114 & 0.092 & 0.040 & 0.021 & $0.122^{*}$ \\
Rule breaking & 0.020 & $0.114^{*}$ & 0.018 & $0.122^{*}$ & 0.110 & $0.159^{* *}$ & $0.127^{*}$ \\
Relapse & 0.009 & -0.034 & 0.016 & 0.028 & -0.006 & $0.118^{*}$ & 0.021 \\
Substance abuse & 0.032 & 0.080 & 0.000 & $0.408^{* *}$ & -0.027 & $0.150^{*}$ & $0.267^{* *}$ \\
Undesirable attitude total & 0.029 & 0.075 & -0.042 & $0.119^{*}$ & 0.043 & $0.125^{*}$ & $0.120^{*}$ \\
\hline
\end{tabular}

Each item of Attitude toward treatment was quantified using a 3-point scale $(1=$ good, $2=$ normal, $3=$ bad). $* p<0.05,{ }^{* *} p<0.01$.

\subsubsection{Comparison of CSs between abstinent and relapsed alcohol-dependent individuals}

The presence of relapse (not occasional re-drinking but continuous re-drinking) within 3 months and 1 year following hospital discharge was investigated through patient interview by the attending physician or deputy. The Family/Social CS of relapsed alcohol-dependent individuals $(n=33)$ was significantly higher than abstinent individuals $(n=176)$ within 3 months $(0.32 \pm 0.25$ vs. $0.23 \pm 0.22$; $\left.{ }^{*} p<0.05\right)$ (Figure 1). Similarly, within 1 year, the Family/Social CS of relapsed individuals $(n=49)$ was significantly higher than abstinent individuals $(n=87)(0.31 \pm 0.23 v s .022 \pm 0.21 ; * p<0.05)$. Although the number of patients who participated in treatment programs plausibly predicts abstinence, it did not impact abstinence within 3 months and 1 year. 
Figure 1. Comparison of CSs between abstinent and relapsed alcohol-dependent individuals.

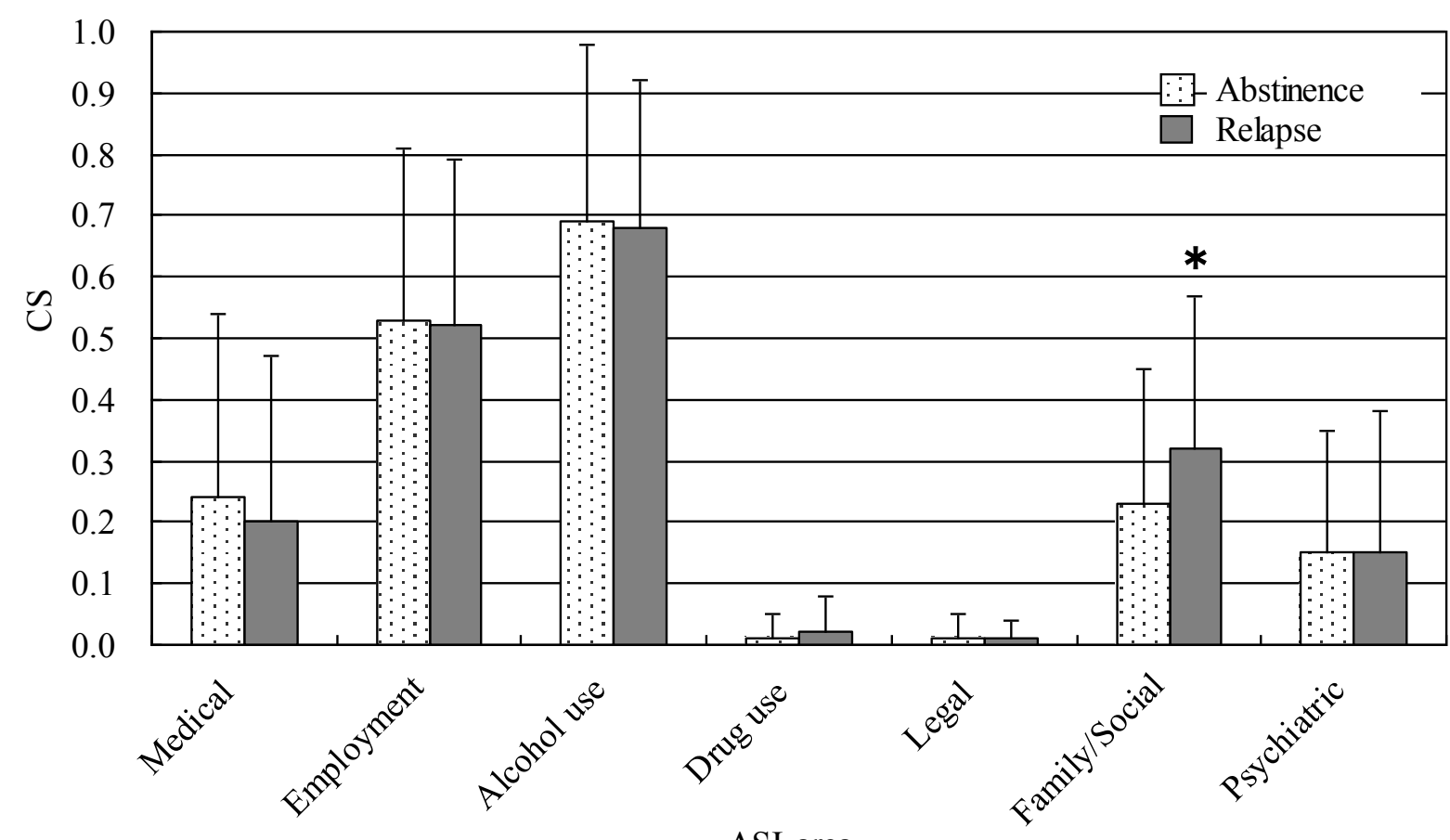

Comparison of CSs between abstinent $(n=176)$ and relapsed $(n=33)$ alcohol-dependent individuals within 3 months $(0.23 \pm 0.22 v s .0 .32 \pm 0.25 ; * p<0.05)$. The follow-up rate was $65 \%$. "Relapse" was defined as continuous re-drinking. Patients with only occasional re-drinking were not included in relapsed patients. The Family/Social CS was significantly different between groups.

\subsection{Comparison between Substance Abuse Groups}

3.3.1. Comparison between alcohol-dependent individuals and drug abusers with individual ASI-J items

Characteristic profiles of alcohol-dependent individuals and drug abusers are shown in Table 3.

(1) Age. The mean (SD) age of the alcohol-dependent individuals (321 patients) was 49.7 (11.0) years, whereas the mean age of drug abusers was 32.9 (9.4) years.

(2) Education and employment. The mean (SD) education of alcohol-dependent individuals was 11.8 (2.7) years. Over $80 \%$ of participants were employed full-time (40 h/week) or part-time during the past 3 years, and $50 \%$ of the participants were receiving a salary in the past 30 days. The mean (SD) education for drug abusers was 11.5 (2.2) years, which was slightly different than alcohol-dependent individuals. The rates of high school and university dropout, however, were higher in drug abusers than in alcohol-dependent individuals. Among drug abusers, only 30\% were employed full-time in the past 3 years, over 35\% were employed part-time, and $25 \%$ were unemployed. The percentage of public assistance recipients in alcohol-dependent individuals was only $8.4 \%$, while the percentage in drug abusers was $21.6 \%$. These results suggest that employment problems were less serious in alcohol-dependent individuals than in drug abusers.

(3) Cohabitant. The marriage rate of alcohol-dependent individuals was $54.2 \%, 45.8 \%$ were living with family (i.e., spouse and at least one child with or without other parents and siblings), and $21.8 \%$ 
were living alone. The states of cohabitation did not change over the past 10 years in $58.9 \%$. For drug abusers, $7.8 \%$ were married, $41.4 \%$ were living with parents, and $80.2 \%$ changed their cohabitant state within the past 10 years.

(4) Abuse. The rate of emotional abuse in alcohol-dependent individuals $(22.2 \%)$ was similar to drug abusers (28.3\%). The rates of physical and sexual assault in alcohol-dependent individuals $(6.9 \%$ and $0.0 \%$, respectively) were low compared with drug abusers ( $28.3 \%$ and $4.4 \%$, respectively).

(5) Use of problematic substance. For alcohol-dependent individuals, the mean (SD) years of alcohol use (i.e., intoxication or feeling improvement) was 20.0 (12.0) years. Regarding voluntary abstinence, $74.8 \%$ relapsed (i.e., consumed alcohol) within the past 3 months. For drug abusers, the mean (SD) years of use of the problem substance was 6.8 (5.7) years, and $40.5 \%$ of the participants relapsed within 3 months. The period of abstinence for alcohol-dependent individuals was shorter than drug abusers, and the rate of alcohol-dependent individuals abstaining from drinking for more than 1 year $(8.7 \%)$ was also significantly less than the rate of drug abusers abstaining from problem substance $(27.6 \%)$.

(6) Psychiatric problems. No significant differences were found in the rate of hospitalization due to psychiatric problems between alcohol-dependent individuals (10.6\%) and drug abusers (16.4\%). The rate of outpatient status due to psychiatric problems was different between alcohol-dependent individuals (11.2\%) and drug abusers (44.8\%). Although the rate of having serious thoughts of suicide in drug abusers was $62.1 \%$, the rate remained at $28.0 \%$ in alcohol-dependent individuals. Whereas $41.4 \%$ of drug abusers actually attempted suicide, $11.5 \%$ of alcohol-dependent individuals attempted suicide. Figure 2 shows that although the lifetime prevalence of psychiatric problems for alcohol-dependent individuals was lower than drug abusers, the rate of psychiatric problems for alcohol-dependent individuals under 40 years old was higher than all alcohol-dependent individuals, with the exception of troubles in understanding, concentrating, or remembering.

(7) Family history. Among alcohol-dependent individuals, $36.3 \%$ of their fathers, $22.6 \%$ of their brothers, $20.9 \%$ of their paternal uncles, and $18.2 \%$ of their grandfathers experienced drinking problems (Figure 3). Compared with drug abusers, alcohol-dependent individuals had more relatives who had significant drinking problems. Regarding parents, the majority of problematic parents of alcohol-dependent individuals were fathers who only had problems with drinking; however, problematic parents of drug abusers consisted of both mothers and fathers who had alcohol, drug, and/or psychiatric problems. 
Table 3. Comparison of profiles between alcohol-dependent individuals and drug abusers.

\begin{tabular}{|c|c|c|c|}
\hline Characteristics & Alcohol-dependent individuals $(n=321)$ & Drug abusers $(n=116)$ & $p$ \\
\hline Sex (\% Males) & 100.0 & 70.0 & \\
\hline Mean age (SD) (years) & $49.7(11.0)$ & $32.9(9.4)$ & $<0.001$ \\
\hline \multicolumn{4}{|l|}{ Education } \\
\hline Mean education (SD) (years) & $11.8(2.7)$ & $11.5(2.2)$ & n.s. \\
\hline$\%$ Junior high school graduate & 29.3 & 23.3 & n.s. \\
\hline$\%$ Some high school & 10.9 & 25.0 & $<0.001$ \\
\hline$\%$ High school graduate & 34.3 & 29.3 & n.s. \\
\hline$\%$ Some college & 3.7 & 11.2 & 0.005 \\
\hline$\%$ College graduate & 18.4 & 9.5 & 0.015 \\
\hline$\%$ Unclear & 3.4 & 1.7 & n.s. \\
\hline \multicolumn{4}{|l|}{ Employment (past 3 years) } \\
\hline$\%$ Full-time & 69.5 & 30.2 & $<0.001$ \\
\hline$\%$ Part-time & 10.6 & 35.3 & $<0.001$ \\
\hline$\%$ Retired & 6.9 & 0.0 & n.s. \\
\hline$\%$ Unemployment & 10.9 & 25.0 & $<0.001$ \\
\hline$\%$ Other & 2.1 & 9.5 & n.s. \\
\hline $\begin{array}{l}\% \text { Public assistance recipient } \\
\text { (past } 30 \text { days) }\end{array}$ & 8.4 & 21.6 & $<0.001$ \\
\hline \multicolumn{4}{|l|}{ Marital status } \\
\hline \% Married & 54.2 & 7.8 & $<0.001$ \\
\hline$\%$ Never married & 21.2 & 67.2 & $<0.001$ \\
\hline$\%$ & 24.6 & 23.3 & n.s. \\
\hline \multicolumn{4}{|l|}{ Separated/Widowed/Divorced } \\
\hline \multicolumn{4}{|l|}{ Cohabitant } \\
\hline \% Family & 45.8 & 12.1 & $<0.001$ \\
\hline \% Spouse & 14.6 & 12.1 & n.s. \\
\hline$\%$ Parents & 13.4 & 41.4 & $<0.001$ \\
\hline$\%$ Alone & 21.8 & 16.4 & n.s. \\
\hline$\%$ Other & 4.4 & 18.0 & n.s. \\
\hline \multicolumn{4}{|l|}{ Years of current cohabitation } \\
\hline$\%$ Within 10 years & 41.1 & 80.2 & $<0.001$ \\
\hline$\% 10-20$ years & 26.5 & 15.5 & 0.010 \\
\hline$\% 20$ years+ & 32.4 & 1.7 & $<0.001$ \\
\hline \multicolumn{4}{|l|}{ Abuse } \\
\hline \% Emotional abuse & 22.2 & 28.3 & n.s. \\
\hline$\%$ Physical abuse & 6.9 & 28.3 & $<0.001$ \\
\hline$\%$ Sexual abuse & 0.0 & 4.4 & 0.001 \\
\hline \multicolumn{4}{|l|}{ Voluntary abstinence } \\
\hline \% Less than 1 month & 47.7 & 19.8 & $<0.001$ \\
\hline \% 1-3 months & 27.1 & 20.7 & n.s. \\
\hline \% 3-6 months & 8.4 & 19.0 & 0.003 \\
\hline$\%$ 6-12 months & 8.1 & 12.9 & n.s. \\
\hline$\% 1-2$ years & 5.0 & 13.8 & 0.003 \\
\hline$\% 2-5$ years & 2.5 & 12.1 & $<0.001$ \\
\hline$\% 5$ years + & 1.2 & 1.7 & n.s. \\
\hline
\end{tabular}

$\mathrm{n}$, number of participants; SD, standard deviation; \% Family of Cohabitant, rate of cohabitation of spouse and at least one child with or without parents, siblings, and relatives. ${ }^{*} p<0.05,{ }^{* *} p<0.01$. 
Figure 2. Comparison of lifetime prevalence of psychiatric problems between alcohol-dependent individuals and drug abusers.

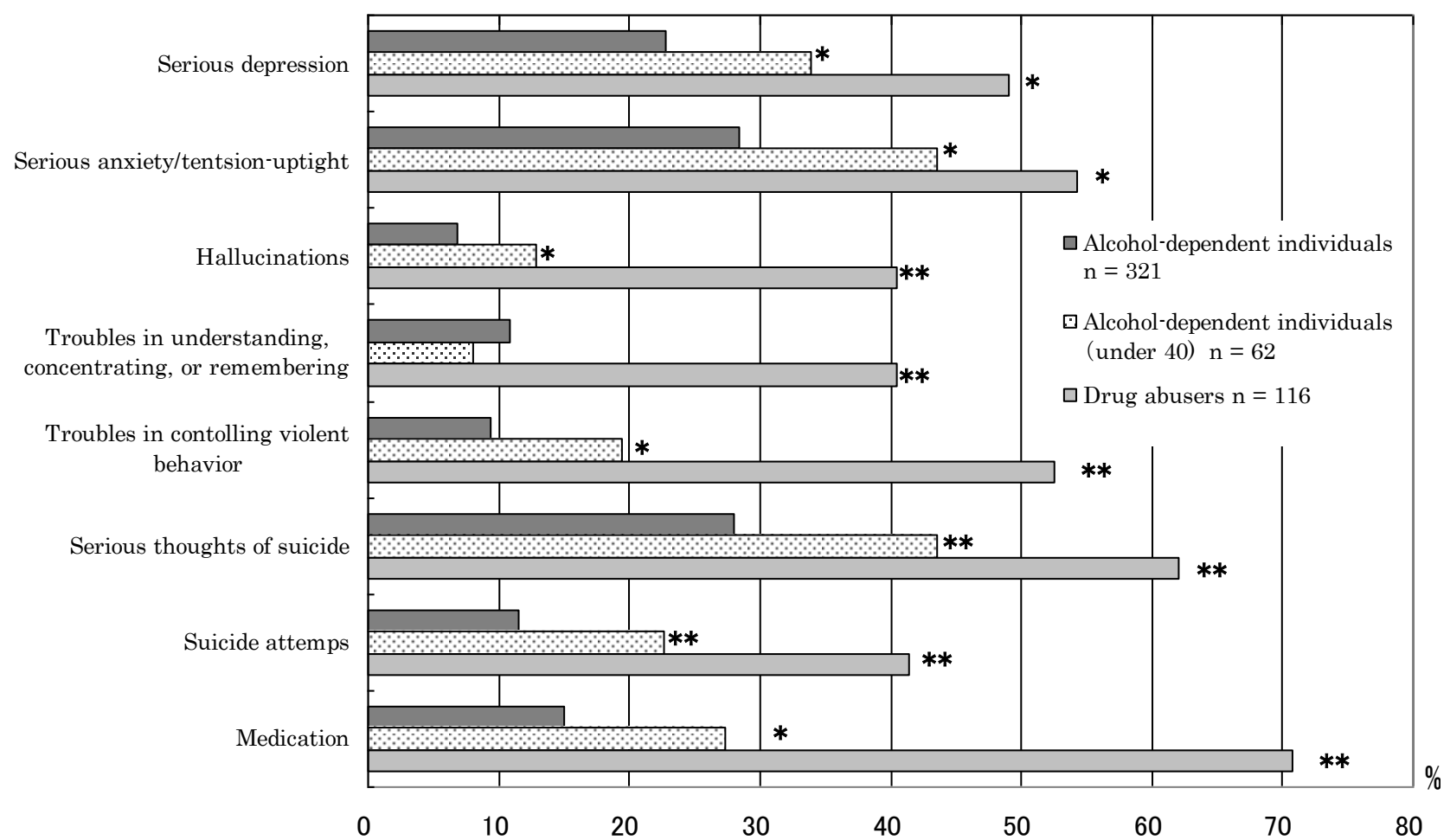

Alcohol-dependent individuals under 40 years old $(n=62)$ were separated from all alcohol-dependent individuals $(n=321)$ and compared because of an apparent difference between the mean age of alcohol-dependent individuals (49.7 years) and drug abusers (32.9 years). All indices showed that alcohol-dependent individuals had experienced fewer psychiatric problems than drug abusers over their lifetime, but alcohol-dependent individuals under 40 years old had experienced more psychiatric problems than all alcohol-dependent individuals, with the exception of troubles in understanding, concentrating, or remembering. Significant differences in each index were examined via the $\chi^{2}$ test $(* p<0.05, * * p<0.01)$.

\subsubsection{Comparison between alcohol-dependent individuals and drug abusers in CS and SR}

The CS and SR of alcohol-dependent individuals were compared with drug abusers [11] (Table 4). A remarkable difference between the CS of alcohol-dependent individuals and drug abusers was seen in the Alcohol use CS (0.55 vs. 0.11), Medical CS (0.24 vs. 0.09), Employment CS (0.54 vs. 0.70), and Psychiatric CS (0.15 vs. 0.31). No major differences were found between alcohol-dependent individuals and drug abusers in the Drug use CS (0.01 vs. 0.10) and Legal CS (0.01 vs. 0.03). No significant difference was observed in the Family/Social CS (0.23 vs. 0.24). Deterioration of medical condition for alcohol-dependent individuals was remarkable compared with drug abusers, while psychiatric problems were relatively unremarkable for alcohol-dependent individuals compared with drug abusers.

The SR indicates more subjective severity than the CS. The SRs of alcohol-dependent individuals and drug abusers generally showed trends similar to CSs, although the SRs in the Drug use area, Legal area, and Family/Social area showed more remarkable differences between alcohol-dependent individuals and drug abusers than corresponding CSs. 
Figure 3. Family history for alcohol-dependent individuals and drug abusers (rate of problematic relatives).

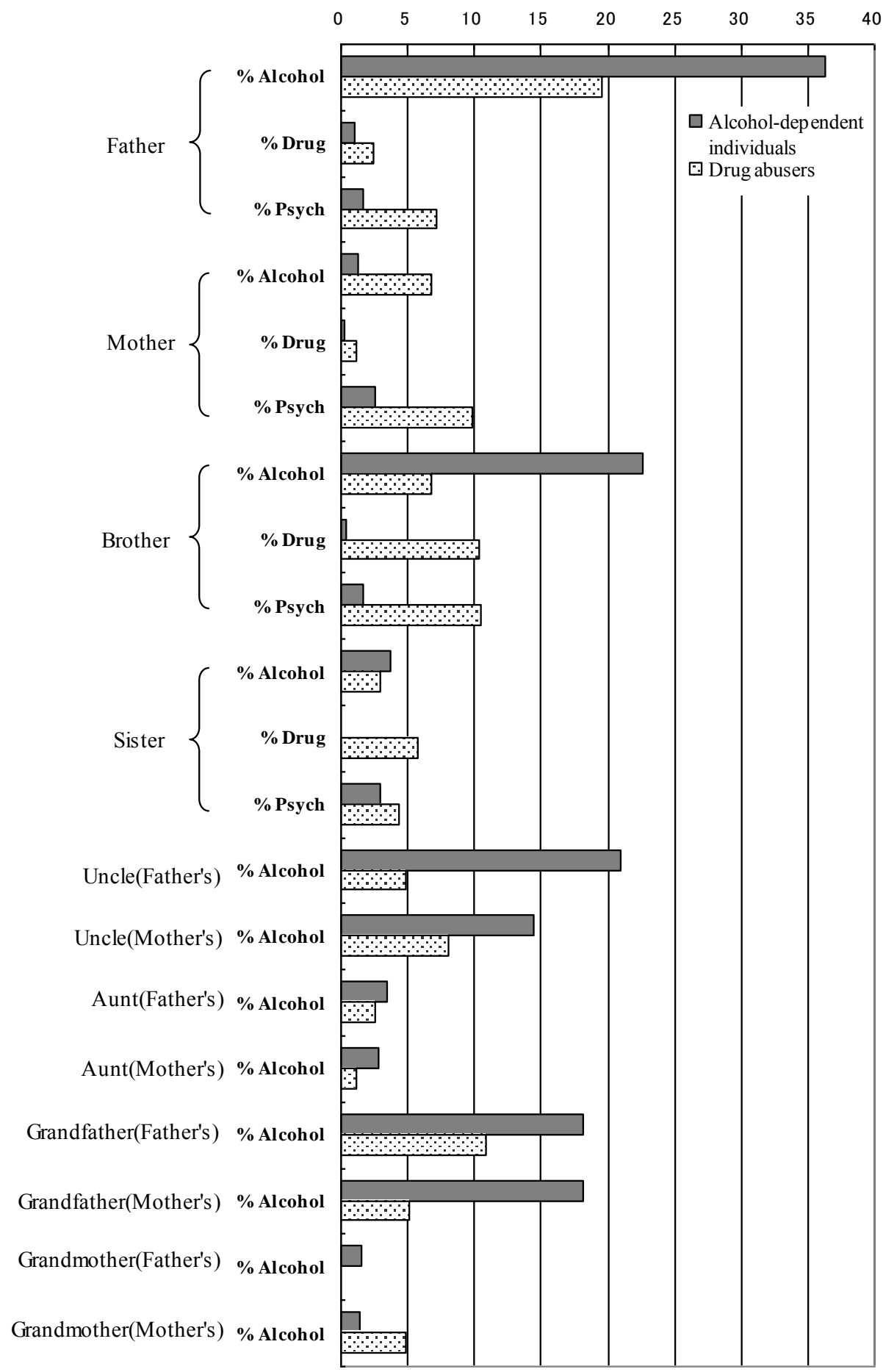

The question in the Family History section of the ASI is, "Have any of your relatives had what you would call a significant drinking, drug use or psych problem - one that did or should have led to treatment?" Uncertain answers ("X" in ASI) and not applicable items ("N" in ASI) were excluded from each number. The items in the boxes indicate relatives of alcohol-dependent individuals $(\%$ Alcohol, rate of problem-drinking relatives; \% Drug, rate of problem drug use relatives; \% Psych, rate of relatives who had some psychiatric problems). Significant differences $(* * p<0.01)$ were found in all indices using the $\chi^{2}$ test. 
Table 4. Comparison between alcohol-dependent individuals and drug abusers in CS, SR, and other related questions.

\begin{tabular}{|c|c|c|c|}
\hline Study country $(n)$ & $\begin{array}{c}\text { Alcohol-dependent } \\
\text { individuals Haraguchi } e t \\
\text { al., Japan }(n=321)\end{array}$ & $\begin{array}{c}\text { Drug abusers } \\
\text { Senoo et al., Japan } \\
\quad(n=111)\end{array}$ & $p$ \\
\hline \multicolumn{4}{|l|}{ Medical } \\
\hline $\mathrm{CS}$ & $0.24(0.30)$ & $0.09(0.21)$ & $<0.001$ \\
\hline SR & $2.41(2.71)$ & $0.69(1.72)$ & $<0.001$ \\
\hline Days of medical problems & $6.67(11.19)$ & $1.83(5.87)$ & $<0.001$ \\
\hline \multicolumn{4}{|l|}{ Employment } \\
\hline $\mathrm{CS}$ & $0.54(0.28)$ & $0.70(0.24)$ & $<0.001$ \\
\hline SR & $3.00(2.81)$ & $5.21(2.86)$ & $<0.001$ \\
\hline Days worked & $11.41(13.01)$ & $6.77(10.55)$ & 0.001 \\
\hline Money earned (1000 yen) & $140.34(204.95)$ & 79.85 (182.86) & 0.005 \\
\hline \multicolumn{4}{|l|}{ Alcohol use } \\
\hline $\mathrm{CS}$ & $0.55(0.22)$ & $0.11(0.19)$ & $<0.001$ \\
\hline SR & $6.20(1.69)$ & $1.39(2.25)$ & $<0.001$ \\
\hline Days drinking & $18.19(12.04)$ & $5.83(10.03)$ & $<0.001$ \\
\hline Days intoxicated & $13.58(12.92)$ & $5.03(9.43)$ & $<0.001$ \\
\hline $\begin{array}{l}\text { Frequency of alcohol delerium tremens } \\
\text { (lifetime) }\end{array}$ & $1.30(3.22)$ & $0.39(1.73)$ & 0.005 \\
\hline \multicolumn{4}{|l|}{ Drug use } \\
\hline $\mathrm{CS}$ & $0.01(0.04)$ & $0.10(0.10)$ & $<0.001$ \\
\hline SR & $0.24(1.19)$ & $5.11(2.94)$ & $<0.001$ \\
\hline Frequency of drug overdose (lifetime) & $0.23(3.00)$ & $1.16(2.44)$ & 0.004 \\
\hline \multicolumn{4}{|l|}{ Legal } \\
\hline $\mathrm{CS}$ & $0.01(0.04)$ & $0.03(0.10)$ & $<0.001$ \\
\hline SR & $0.10(0.66)$ & $0.54(1.54)$ & $<0.001$ \\
\hline $\begin{array}{r}\text { Charges resulting in convictions } \\
\text { (lifetime) }\end{array}$ & $0.14(0.74)$ & $1.00(1.57)$ & 0.044 \\
\hline \multicolumn{4}{|l|}{ Family \& Social } \\
\hline $\mathrm{CS}$ & $0.23(0.21)$ & $0.24(0.23)$ & n.s. \\
\hline SR & $2.77(2.87)$ & $3.66(2.63)$ & 0.003 \\
\hline Days of conflicts w/family & $5.57(10.24)$ & $2.53(6.91)$ & 0.004 \\
\hline Days of conflicts w/others & $2.72(7.96)$ & $2.20(5.97)$ & n.s. \\
\hline \multicolumn{4}{|l|}{ Psychiatric } \\
\hline $\mathrm{CS}$ & $0.15(0.20)$ & $0.31(0.27)$ & $<0.001$ \\
\hline SR & $1.84(2.39)$ & $3.85(3.33)$ & $<0.001$ \\
\hline Days of psychological Problems & $5.68(10.58)$ & $10.10(13.00)$ & $<0.001$ \\
\hline
\end{tabular}


3.3.3. Comparison of CS in Japanese alcohol-dependent individuals with CS in alcohol-dependent individuals in other countries

The present CSs were compared with those in alcohol-dependent individuals in the United States, Switzerland, The Netherlands, and Germany (Figure 4). Almost all data were collected from facilities that specialized in addiction treatment (refer to legend of Figure 4). The average ages (SD) of the participants from four countries (data unknown from The Netherlands) ranged from 40 (8) to 50 (11) years. Participants from the United States, Germany, and Japan were male only, and participants from Switzerland and The Netherlands comprised 78 men and 22 women, and 101 men and 43 women, respectively. The Japanese Alcohol use CS (0.67) was comparable to The Netherlands CS (0.79). The Psychiatric CS (0.15) was the lowest of the five CSs. The Netherlands CSs were the highest in the five areas (Medical, Alcohol use, Legal, Family/Social, and Psychiatric areas). The United States CS was the highest in the Employment area, and the Drug use CS was high compared with other countries.

Figure 4. Differences between Japanese data and other data.

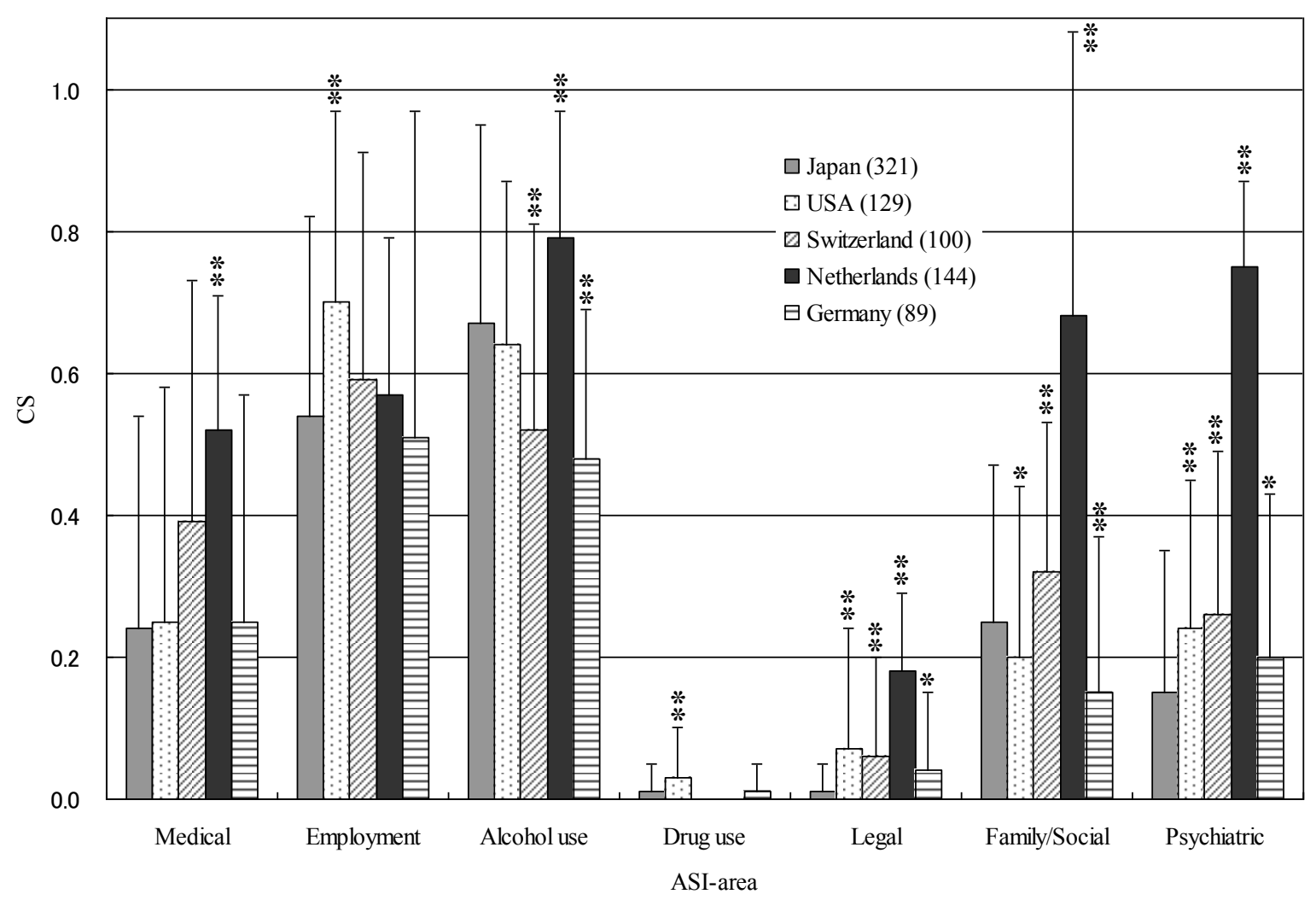

Differences between Japanese data and other data [5-7,9] were analyzed by Welch's t-test $(* p<0.05$, $* * p<0.01)$. Vertical bars indicate the standard deviation. Numbers in parentheses are the numbers of subjects. Japanese participants (male inpatients only) were recruited from nine hospitals specializing in addiction treatment. The average number of years of education (SD) was 11.8 (2.7), 54.2\% were married, and $80.1 \%$ were employed (full-time and part-time in the previous 3 years). United States data (male only) were drawn from admissions data in public and private, inpatient, outpatient, or partial hospital treatment programs in the Philadelphia area. The average number of years of 
education (SD) was 12.1 (2). Swiss participants (78 men and 22 women) were recruited from four institutions in the Lausanne area, a French-speaking region in Western Switzerland, 30\% were married, and 62\% were employed. Participants from The Netherlands (101 men and 43 women) were patients admitted to the diagnostic unit of the institute for addiction treatment, a center for the clinical treatment of drug and alcohol addicts, 35.4\% were married, and 34\% were employed. Participants from Germany were all inpatients (male only) requesting treatment for alcohol dependence, and $38.4 \%$ were married. Although we used the above United States data [5] because these were male data, a report [3] shows data for 1935 alcohol-dependent patients (both males and females) in the United States as the following: Medical CS (0.14), Employment CS (0.55), Alcohol use CS (0.29), Drug use CS (0.07), Legal CS (0.21), Family/Social CS (0.10), and Psychiatric CS (0.15).

\subsection{Internal Consistency and Concurrent Validity}

To examine the internal consistency of the CSs in the seven areas, Cronbach's alpha coefficients were calculated (Table 5). The coefficients ranged from 0.84 in the Psychiatric area to 0.53 in the Family/Social area. Overall, internal consistency of the ASI-J area was acceptable, with the exception of the Family/Social area. Regarding correlation coefficients between the component items of the Family/Social CS, the satisfaction rate for married life and days with trouble within family minimally correlated with other items (-0.03 to 0.22$)$.

Because both the CS and SR assess the level of subject function in a given problem area, examining whether they offer correlated measurements of subject function in each of the problem areas is important [11]. The methods of deriving the CS and SR are based on both similar and different questions within each area. Significant positive correlations between the CS and SR were observed in all problem areas. CS-SR relationships were strong in the Medical area (0.69), Psychiatric area (0.66), and Drug use area (0.62), while weak in the Alcohol use area (0.22) and Employment area (0.29).

The CS and SR in the Alcohol use area were weakly but significantly correlated with some of the biological markers (Table 6). Correlation coefficients between the Alcohol use CS and GOT at hospitalization, between the Alcohol use CS and GPT at hospitalization, and between the Alcohol use CS and $\gamma$-GTP at hospitalization were $0.23\left({ }^{*} p<0.01\right), 0.17\left({ }^{*} p<0.01\right)$, and $0.25(* p<0.01)$, respectively. Correlation coefficients between the Alcohol use SR and GOT at hospitalization and between the Alcohol use SR and GPT at hospital discharge were $0.13\left({ }^{*} p<0.05\right)$ and $0.17\left({ }^{*} p<0.01\right)$, respectively. These results partially support the concurrent validity of the Alcohol use CS and SR. 
Table 5. Internal consistency of CSs in each ASI-J area and correlations between CSs and SRs in alcohol-dependent individuals $(n=321)$.

\begin{tabular}{|c|c|c|}
\hline ASI-J area & $\begin{array}{c}\text { Cronbach's } \alpha \text { (Number of } \\
\text { CS items [SD]) }\end{array}$ & $\begin{array}{c}\text { Correlation between CS } \\
\text { and SR }\end{array}$ \\
\hline Medical & $0.794(3)$ & $0.688 * *$ \\
Employment & $0.667(4)$ & $0.290 * *$ \\
Alcohol use & $0.671(6)$ & $0.217 * *$ \\
Drug use & $0.700(17)$ & $0.623 * *$ \\
Legal & $0.712(5)$ & $0.520 * *$ \\
Family/Social & $0.534(5)$ & $0.584 * *$ \\
Psychiatric & $0.836(1)$ & $0.664 * *$ \\
\hline
\end{tabular}

$* * p<0.01$.

Table 6. Correlation coefficients between the Alcohol use CS and biological markers $(n=321)$.

\begin{tabular}{|c|c|c|c|c|}
\cline { 2 - 5 } \multicolumn{1}{c|}{} & $\begin{array}{c}\text { GOT (at } \\
\text { hospitalization) }\end{array}$ & $\begin{array}{c}\text { GPT (at } \\
\text { hospitalization) }\end{array}$ & $\begin{array}{c}\gamma \text {-GTP (at } \\
\text { hospitalization) }\end{array}$ & $\begin{array}{c}\text { GPT (at hospital } \\
\text { discharge) }\end{array}$ \\
\hline Alcohol use CS & $0.233 * *$ & $0.169 * *$ & $0.245 * *$ & 0.066 \\
\hline Alcohol use SR & $0.129 *$ & 0.061 & 0.007 & $0.168 * *$ \\
\hline
\end{tabular}

GOT, glutamic oxaloacetic transaminase; GPT, glutamic pyruvic transaminase; $\gamma$-GTP, $\gamma$-glutamyl transpeptidase. ${ }^{*} p<0.05,{ }^{* *} p<0.01$.

\section{Discussion}

\subsection{Usefulness of the ASI-J in Customized Treatment}

The measure of independence of the problem areas illustrates the ability of the ASI-J to classify and quantify wide-ranging problems in addition to alcohol use problems to enable customized treatment. As shown in Table 1, the CSs in ASI areas show relatively few relationships with each other. These data substantiated the relative independence of the problem areas and the ability of the questions to assess these various problems demonstrated in other countries [1,6,7,13-15].

Although each area of the ASI-J is basically independent, the Psychiatric CS in the ASI-J was slightly but significantly related with the Family/Social CS and Drug use CS and Alcohol use CS. This trend was similar to overseas data $[1,14,16]$. Kosten et al. [14] indicated that most addicts with psychological problems had poor social adjustment and problems in the other ASI areas, suggesting that one or more subgroups of multiproblem addicts who have a variety of psychiatric disorders might be identified by the ASI. The ASI-J would be useful for identifying patients who need early concurrent treatments by determining relationships between their Psychiatric CS and other CSs.

We underscore the function of Family History for customized treatment. Cotton [17] (a review of 32 studies researching frequency of paternal alcoholism) reported a $27 \%$ incidence of alcoholism in the fathers of 4329 alcohol-dependent individuals. These data were obviously higher than the rates of alcoholism expected in the general population (2-3\%) and in males over 40 years old (6-10\%) [18]. 
Our results showing that problem-drinking was present in $36 \%$ of 302 subjects' fathers and present in $19 \%$ of 88 drug abusers' fathers (Figure 3) were nearly consistent with Cotton's results. These rates were higher than the general population and higher than the rates reported in Cotton's study. Furthermore, a study by Iwakura [19] indicated that subjects who grew up in dysfunctional families (e.g., with parents who had alcohol-related problems) struggled with more complicated treatment issues. Therefore, clinicians should thoroughly grasp the patients' psychosocial histories and provide proper treatment and support. Alcohol dependence in parents has often been assessed by the Children of Alcohol-dependent individuals Screening Test (CAST), which consists of 30 questions. The present results suggest that simple questions in the ASI Family History section, rather than the CAST, are useful for identifying adult children of dysfunctional families with alcoholic problems and assessing the needs for additional treatment.

\subsection{Usefulness of ASI-J as a Prediction Tool}

Drug use, Psychiatric, or Family relationship problems in subjects were related with total undesirable attitude toward treatment (Table 2). Additionally, subjects who had employment problems occasionally lacked cooperation with other inpatients and broke the rules of treatment. The family, criminal, employment, and psychological problems seen among alcohol and drug abuse patients have reportedly been important predictors of response to treatment, with psychiatrically ill and especially antisocial substance abusers particularly likely to show poor treatment response and early relapse, regardless of the treatment modality or setting [20,21]. Our results are consistent with these reports. The moderate relationship between the Drug use CS at the start of hospitalization and relapse during treatment (temporarily leaving the hospital and staying out overnight) suggests difficulty in maintaining abstinence from drug use when the patient is temporarily away from the hospital. For successful hospitalization, improving psychiatric problems as early as possible after hospitalization, controlling family relationships, and providing guidance for staying away from the problem substance during temporary retreats away from the hospital are very important. As McLellan et al. indicated [3], additional services for addiction-related problems at an early stage will improve the outcomes of standard addiction treatments.

Figure 1 shows that the Family/Social CSs at hospitalization were significantly different between subjects who relapsed within 3 months after leaving the hospital and subjects who continued to abstain from drinking. Subjects who had serious family and social relationship problems tended to relapse, suggesting that control of interpersonal relationships during treatment is a key predictor of response to treatment. In contrast, Alcohol use CSs at hospitalization were not effective predictors of relapse and compliance to treatment. These results, together with the results in Table 2, support the view that useful predictors of relapse and compliance to treatment are not the serious drinking itself, but rather the various CSs of the ASI-J at hospitalization, although more data are necessary to confirm these relatively weak relationships. 


\subsection{Usefulness of the ASI-J as a Comparison Tool}

We tested the usefulness of the ASI-J as a comparison tool to reveal features of the analyzed groups. Argeriou et al. [13] proved sensitivity of the ASI to detect differences in ASI scores across various subgroups (i.e., homeless, near homeless, white, black, Hispanic, men, and women). Our study demonstrated the usefulness of the ASI-J in finding differences in alcohol-dependent individuals in Japan from both drug abusers in Japan and alcohol-dependent individuals in other countries.

\subsubsection{Features of alcohol-dependent individuals: comparison with drug abusers}

(1) Seriousness of medical problem. Deterioration of medical condition for alcohol-dependent individuals was remarkable compared with drug abusers. Various medical complications attributable to a longer duration of problematic consumption may underlie the deterioration of medical condition.

(2) High rate of employment. The rate of employment (full-time or part-time during the past 3 years) of alcohol-dependent individuals was higher $(80.1 \%)$ and the rate receiving public assistance was lower $(8.4 \%)$ than drug abusers $(65.5 \%$ and $21.6 \%$, respectively). Economic crises do not appear to occur often in alcohol-dependent individuals compared with drug abusers. The first possible reason for this high rate of employment might be a short clinical history of Japanese alcohol-dependent individuals. Although the present study subjects $(n=321)$ were all inpatients, $60 \%$ were first hospitalizations, and the average frequency of hospitalization was two times, suggesting that these individuals basically lived without uncontrollable failure prior to hospitalization. The second possible reason might be subject selection. In the present study, we selected subjects who were able to properly answer the ASI-J questions. This selection might be too restrictive to represent all Japanese alcoholic individuals.

(3) Difficulty in voluntary abstinence. In Japan, alcohol is not prohibited. Moreover, a favorable climate has existed for alcohol use since ancient times [2]. We found that nearly half of subjects relapsed within 1 month after completing inpatient treatment, 75\% resumed drinking within 3 months, and over $90 \%$ resumed drinking within 1 year. The relapse rates are remarkably high, considering that alcohol dependence treatment in Japan is generally planned with the goal of achieving abstinence. However, $60 \%$ and $28 \%$ of drug abusers continued voluntary abstinence within 3 months and over 1 year, respectively, suggesting that abstention from drinking is very difficult because of poor deterrence.

(4) Unchanging family structure. Nearly half of drug abusers lived with parents, possibly because they were not employed due to serious problems of addiction. In contrast, nearly half of the alcohol-dependent individuals lived with families (spouse and children) who provided for them, and their cohabitant states remained unchanged for 10-20 years. The divorce rate among those who were married was lower in alcohol-dependent individuals than in drug abusers (30\% vs. 75\%). These results suggest that alcohol dependence can deteriorate in unchanging family structures. Saito [22] reported that for patients who cohabitate with families, the treatment approach for the families needs to be assessed to determine the functional and emotional roles played by family members. Improving family education and family therapy may be indispensable for successful addiction treatment.

(5) Psychiatric problems in young alcohol-dependent individuals. Regarding psychiatric problems, alcohol-dependent individuals were relatively not serious compared with drug abusers. However, 
Figure 3 shows that the prevalence of all psychiatric problems in alcohol-dependent individuals who were less than 40 years old were significantly high compared with older subjects, with the exception of difficulty in understanding, concentrating, or remembering. These results support the view that careful assessment of psychiatric treatment is needed for young alcohol-dependent individuals.

To summarize, the most obvious difference is a better environment around alcohol-dependent individuals compared with drug abusers. Their states of alcohol dependence gradually deteriorated in unchanging family structures and conditions of employment. Families should understand the family relationships and alcohol dependence. Furthermore, a serious problem is that treatment efficacy has been low because long-term abstinence is very difficult. Continued abstention from drinking, even with psychological education and family cooperation, is still difficult because drinking alcohol is generally a daily custom.

\subsubsection{Comparison of CSs of alcohol-dependent individuals among studies}

The present CSs of each area were compared with overseas data (Figure 4). Although information was limited for circumstances of treatment instruments and nationality in each country, we found some similarities and differences in each country's data. In the Japanese data, although the Alcohol use CS was relatively high, the Psychiatric CS was the lowest of the five datasets. These data, however, may indicate that the CSs are little different among countries, with the exception of The Netherlands which had the highest scores in the five areas.

Through the various comparisons of ASI data, treatment facilities may be able to discuss and exchange information about treatment techniques and methods in view of similarities and differences of patient features and their treatment environments. Furthermore, the usefulness of the ASI as a comparison tool will be supported by the accumulation of data from normal individuals and nondependent individuals with alcohol problems. In a study of the German version of the European ASI, differences were observed between groups of patients with and without a diagnosis of alcohol dependence [9].

\subsection{Limitations and Further Study}

Although our data illustrated the ability of the ASI to assess multidimensionally addiction-related problems, the actual numeric value of the CS has no intrinsic meaning [23]. Moreover, CSs are not similarly scaled and therefore cannot be compared between problem areas [13]. In the present study, cutoff points of CSs could not be estimated because the ranges of CSs, especially the Drug use and Legal CSs, were not wide. Additionally, insufficient data exist to definitively know the normal range. The standard of severity in each area is not precisely known, although cutoff points of the Alcohol use CS and Drug use CS (0.17 and 0.16, respectively) have been provided [24]. Therefore, we need to refer to the SR to determine the priority of treatment in seven problem areas, although the SR is a subjective rating and is viable as a clinical summary only for initial treatment planning and referral [5]. Further accumulation of normal data is also needed to define the numerical meaning of the CS.

We compared domestic and overseas CS data for alcohol-dependent individuals. Although these non-comprehensive data showed roughly the features of each country's data, we could not understand 
the precise reasons for differences only though CS comparisons. Further study of nationality and the features of treatment facilities would be necessary. International joint investigations of alcohol-dependent individuals in similarly controlled settings using the ASI as a common interview may reveal the features of nationality and facilities in detail.

Regarding the ASI as a prediction tool, although this study suggests its usefulness, further research will be needed, considering the low correlations between CSs and relapse and compliance to treatment in addition to the low follow-up rate $(65 \%)$ for the data at 3 months post-discharge in the present study.

Finally, the reliability and validity of the ASI-J in alcohol-dependent individuals were not sufficiently examined in the present study. The ASI-J demonstrated good internal consistency, with the exception of the Family/Social area, and criterion validity measured correlations between the Alcohol use CS/SR and biological markers. These results suggest that the Alcohol use CS may be an index for determining severity and reflecting recent alcohol use. Although our team already substantiated inter-rater reliability through training for administration of the ASI and concurrent validity of the ASI-J in drug abusers, we did not examine these variables in alcohol-dependent individuals.

\section{Conclusions}

This study demonstrated that the ASI-J is useful for planning customized treatment. The ASI-J served as a predictive tool for relapse and compliance to treatment and was shown to be useful as a comparison tool to clarify similarities and differences between substance abuser groups. The present data may contribute to accumulation of the international ASI database.

\section{Acknowledgements}

We are grateful to the subjects for their participation in this study. We are also grateful to Ms. Yoko Ishibashi and Ms. Fumiyuki Chin for fruitful discussion and the late Hitomi Yamamoto for filing data. We acknowledge Mr. Michael Arends for his assistance with editing the manuscript. This work was supported by grants from the Ministry of Education, Culture, Sports, Science and Technology of Japan (17025054), the Ministry of Health, Labour and Welfare of Japan (H17-Pharmaco-001, H19-Iyaku-023), the Japan Society for the Promotion of Science (quasi-governmental organization; 17730421, 17591238), and The Mitsubishi Foundation.

\section{References}

1. Hendriks, V.M.; Kaplan, C.D.; van Limbeek, J.; Geerlings, P. The Addiction Severity Index: reliability and validity in a Dutch addict population. J. Subst. Abuse Treat. 1989, 6, 133-141.

2. Shirakura, K. Introduction: Substance-Related Disorders. In Encyclopedia of Clinical Psychiatry [Rinsho Seishin Igaku Koza], Vol. 8; Matsushita, M., Asai, M., Sato, M., Suwaki, H., Eds.; Nakayamashoten: Tokyo, Japan, 1999; pp. 100-107. 
3. McLellan, A.T.; Cacciola, J.S.; Alterman, A.I.; Rikoon, S.H.; Carise, D. The Addiction Severity Index at 25: origins, contributions and transitions. Am. J. Addict. 2006, 15, 113-124.

4. McLellan, A.T.; Luborsky, L.; Woody, G.E.; O'Brien, C.P. An improved diagnostic evaluation instrument for substance abuse patients: The Addiction Severity Index. J. Nerv. Ment. Dis. 1980, 168, 26-33.

5. McLellan, A.T.; Kushner, H.; Metzger, D.; Peters, R.; Smith, I.; Grissom, G.; Pettinati, H.; Argeriou, M. The fifth edition of the addiction severity index. J. Subst. Abuse Treat. 1992, 9, 199-213.

6. Daeppen, J.B.; Burnand, B.; Schnyder, C.; Bonjour, M.; Pécoud, A.; Yersin, B. Validation of the Addiction Severity Index in French-speaking alcoholic patients. J. Stud. Alcohol 1996, 57, 585-590.

7. Dejong, C.A.; Willems, J.C.; Schippers, G.M.; Hendriks, V.M. The Addiction Severity Index: reliability and validity in a Datch alcoholic population. Int. J. Addict. 1995, 30, 605-616.

8. McLellan, A.T.; Luborsky, L.; Cacciola, J.; Griffith, J.; Evans, F.; Barr, H.L.; O'Brien, C.P. New data from the Addiction Severity Index: reliability and validity in three centers. J. Nerv. Ment. Dis. 1985, 173, 412-423.

9. Scheurich, A.; Müller, M.J.; Wetzel, H.; Anghelescu, I.; Klawe, C.; Ruppe, A.; Lörch, B.; Himmerich, H.; Heidenreich, M.; Schmid, G.; Hautzinger, M.; Szegedi, A. Reliability and validity of the German version of the European Addiction Severity Index (EuropASI). J. Stud. Alcohol. 2000, 61, 916-919.

10. Mäkelä, K. Studies of the reliability and validity of the Addiction Severity Index. Addiction 2004, 99, 398-418.

11. Zanis, D.A.; McLellan, A.T.; Cnaan, R.A.; Randall, M. Reliability and validity of the Addiction Severity Index with a homeless sample. J. Subst. Abuse Treat 1994, 11, 541-548.

12. Senoo, E.; Ogai, Y.; Haraguchi, A.; Kondo, A.; Ishibashi, Y.; Umeno, M.; Kikumoto, H.; Hori, T.; Komiyama, T.; Kato, R.; Aso, K.; Asukai, N.; Wada, K.; Saitoh, S.; Ikeda, K. Reliability and validity of the Japanese version of the Addiction Severity Index (ASI-J). Nihon Arukoru Yakubutsu Igakkai Zasshi 2006, 41, 368-379.

13. Argeriou, M.; McCarty, D.; Mulvey, K.; Daley, M. Use of the Addiction Severity Index with homeless substance abusers. Subst. Abuse Treat. 1994, 11, 359-365.

14. Kosten, T.R.; Rounsaville, B.J.; Kleber, H.D. Concurrent validity of the Addiction Severity Index. J. Nerv. Ment. Dis. 1983, 171, 606-610.

15. Krenz, S.; Dieckmann, S.; Favrat, B.; Spagnoli, J.; Leutwyler, J.; Schnyder, C.; Daeppen, J.B.; Besson, J. French version of the Addiction Severity Index (5th edition): validity and reliability among Swiss opiate-dependent patients. French validation of the Addiction Severity Index. Eur. Addict. Res. 2004, 10, 173-179.

16. Hodgins, D.C.; El-Guebaly, N. More data on the Addiction Severity Index: reliability and validity with the mentally ill substance abuser. J. Nerv. Ment. Dis. 1992, 180, 197-201.

17. Cotton, N.S. The familial incidence of alcoholism: a review. J. Stud. Alcohol. 1979, 40, 89-116.

18. Saito, S. Marital interaction and the transmission across generations in alcoholic families. Seishin Shinkeigaku Zasshi 1988, 90, 717-748. 
19. Iwakura, N.; Sera, M.; Yonezawa, H.; Juukuroki, H.; Shingai, N. A study on the relationship between alcoholics and alcohol-related problems of the parental generation. Jpn. J. Addict. Fam. 2006, 23, 55-63.

20. Rounsaville, B.J.; Dolinsky, Z.S.; Babor, T.F.; Meyer, R.E. Psychopathology as a predictor of treatment outcome in alcoholics. Arch. Gen. Psychiat. 1987, 44, 505-513.

21. Woody, G.E.; McLellan, A.T.; Luborsky, L.; O'Brien, C.P. Sociopathy and psychotherapy outcome. Arch. Gen. Psychiatry 1985, 42, 1081-1086.

22. Saito, T. Trends in therapy for alcoholism. Seishin Shinkeigaku Zasshi 2007, 109, 536-540.

23. McGahan, L.P.; Griffith, A.J.; McLellan, A.T. Composite scores from the Addiction Severity Index: Manual and Computer Software. Veterans Administration Press: Philadelphia, PA, USA, 1986.

24. Rikoon, S.H.; Cacciola, J.S.; Carise, D.; Alterman, A.I.; McLellan, A.T. Predicting DSM-IV dependence diagnoses from Addiction Severity Index composite scores. J. Subst. Abuse Treat. 2006, 31, 17-24.

(C) 2009 by the authors; licensee Molecular Diversity Preservation International, Basel, Switzerland. This article is an open-access article distributed under the terms and conditions of the Creative Commons Attribution license (http://creativecommons.org/licenses/by/3.0/). 